\title{
First Detection of a Frequency Multiplet in the Line Profile Variations of RR Lyrae: Towards an Understanding of the Blazhko Effect ${ }^{1}$
}

\author{
K. Kolenberg ${ }^{2}$, C. Aerts ${ }^{3}$ \\ Instituut voor Sterrenkunde, Katholieke Universiteit Leuven, Belgium \\ M. Chadid \\ European Southern Observatory, Alonso de Cordova, 3107, P.O. 19001, \\ Santiago, Chile \\ D. Gillet
}

Observatoire de Haute-Provence, Saint-Michel-l'Observatoire, F-04870, France

\begin{abstract}
We provide the first detection of a frequency multiplet in the line profile variations of RR Lyrae. Performing a period analysis on 669 high resolution line profiles obtained with the spectrograph ELODIE at OHP, we clearly detect a multiplet structure, with a separation equal to the Blazhko frequency, around the main frequency and its harmonics. The triplet components are very prominent; additional observations are needed to decide about the existence of a quintuplet. The complete understanding of the origin of the Blazhko effect still needs further theoretical modelling and better observations. Our detection of the frequency multiplet in the line profile variations is a first essential step towards a decisive confrontation between the theoretical models and the observations.
\end{abstract}

\section{Introduction}

It is well-known that $R R$ Lyrae stars play a very significant role in astrophysics, not only because they are standard candles, but also because they are witnesses of the evolution of the universe at young age. At the same time, they are considered to be prototypes of pure radial pulsators. Although these stars are well-studied, major questions remain to be solved, such as the origin of the socalled Blazhko effect, which is shown by $20-30 \%$ of the RRab stars (Szeidl \& Kolláth 2000).

Different hypotheses are formulated to explain the amplitude modulation. Most speculations refer to two types of models, which both involve nonradial modes (Kovács 1994 \& 1995):

\footnotetext{
${ }^{1}$ Based on observations obtained at the Haute-Provence Observatory in France

${ }^{2}$ Aspirant Fellow, Fund for Scientific Research, Flanders

${ }^{3}$ Postdoctoral Fellow, Fund for Scientific Research, Flanders
} 
- The resonance models, based on a (nonlinear) resonance between the radial fundamental mode and a nonradial mode. (Dynamical interaction - Van Hoolst, Dziembowski, \& Kawaler 1998; Van Hoolst 2000; Steady resonant pulsation - Cox 1993.)

- The magnetic models, like the oblique pulsator model which assumes that the Blazhko stars have a magnetic field which is oblique to the pulsation axis (a replica of the model proposed by Kurtz (1982) for the roAp stars - see also Cousens (1983), Shibahashi \& Takata (1995), and Shibahashi $(2000)$.

Up to now, nonradial components have not been found explicitly in the observational studies. High resolution spectral line profiles are a much better diagnostic to find and identify these modes. In this respect, RR Lyrae, by far the brightest Blazhko star and with a considerable amplitude difference, is the most suitable object to detect them.

\section{Observations}

RR Lyrae was measured with ELODIE, the cross-dispersed spectrograph attached to the 1.93-m telescope of the Observatoire de Haute-Provence, resulting in 669 spectra with $S / N>200$ and a time resolution less than $5 \%$ of the radial pulsation period ( $13 \mathrm{~h} 36 \mathrm{~min})$. The observations were obtained during 16 nights, covering a time-span of $433 \mathrm{~d}$. Six complete pulsation cycles, each observed during two or three consecutive nights, have been monitored. Certain phases in the Blazhko cycle $(40.8 \mathrm{~d})$ were not covered.

In our study, we have used the profile of the Fe II $\lambda 4923.921 \AA$ line. For a detailed description of the data reduction see Chadid \& Gillet (1997).

\section{Results of the Period Analyses}

Analyses of the first moment with PDM (Stellingwerf 1978) and CLEAN (Roberts, Lehár, \& Dreher 1987) always reveal the radial pulsation frequency, $f_{\mathrm{P}}$, as the principal frequency. Two further observations are striking:

- The nonlinear character of the pulsation is derived from the spectra. We observe the harmonics of $f_{\mathrm{P}}$, surpassing the noise level until $8 f_{\mathrm{P}} . f_{\mathrm{P}}, 2 f_{\mathrm{P}}$ and $3 f_{\mathrm{P}}$ are the highest three peaks in the periodogram.

- We detect a multiplet structure, with a separation equal to the Blazhko frequency, around $f_{\mathrm{P}}$ and its harmonics. We observe two maxima around the main peak, very close to the expected positions of the triplet components $f_{\mathrm{P}}-f_{\mathrm{B}}$ and $f_{\mathrm{P}}+f_{\mathrm{B}}$ (see Fig. 1). They are aliases of the exact triplet frequencies, caused by the time spacing of the data. The triplet frequencies are the highest peaks after $f_{\mathrm{P}}, 2 f_{\mathrm{P}}$ and $3 f_{\mathrm{P}}$. We always observe the left side-peak $f_{\mathrm{P}}-f_{\mathrm{B}}$ to have a higher amplitude than $f_{\mathrm{P}}+f_{\mathrm{B}}$. Also, the higher order harmonics of $f_{\mathrm{P}}$ show this triplet structure, but in a less obvious way since their amplitudes are smaller. 


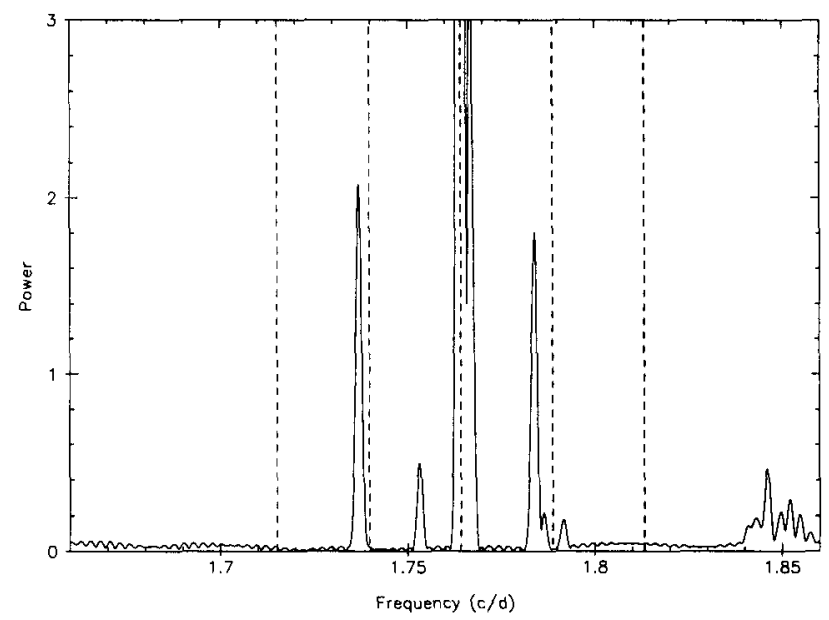

Figure 1. The multiplet structure, centered around the main frequency, of a CLEAN analysis on the first moment. Dashed vertical lines indicate the expected positions of the quintuplet components.

Period analyses of the second and third order moments also reveal the multiplet structure. The Blazhko frequency itself appears in the periodogram of the equivalent width.

A two-dimensional CLEAN analysis, carried out on the normalized flux of each wavelength bin across the absorption line, gives summed periodograms that are less noisy. Again we observe the triplet structure over a large part of the profile. The side-peak $f_{\mathrm{P}}+f_{\mathrm{B}}$ is relatively much lower in this two-dimensional analysis.

The existence of the quintuplet structure, predicted by the oblique pulsator model, remains uncertain. We undoubtedly observe a triplet structure around $f_{\mathrm{P}}$ and its harmonics. In varying the parameters of the frequency search codes, we find some indication for the presence of the quintuplet frequencies. In about $50 \%$ of the cases they are hidden in the noise and in alias frequencies. A better coverage of the Blazhko cycle is needed to draw more definitive conclusions about the occurrence of the quintuplet.

\section{Conclusions and Future Work}

Our detection of a frequency multiplet is the first detailed spectroscopic confirmation of amplitude modulation in the line profiles of RR Lyrae. Since all currently accepted models for explaining the Blazhko effect involve nonradial components, this provides a strong indication for the presence of nonradial modes in Blazhko stars.

The next step in our research will consist in deriving a nonlinear generalization of the moment method (Aerts, De Pauw, \& Waelkens 1992), which will lead to an identification of the degree of the pulsation components corresponding to 
the side-peak frequencies. This degree will put constraints upon the validity of the models.

Furthermore, we will explore the theoretical models. On the one hand, a refinement of the magnetic model for RR Lyrae, taking into account nonlinear terms up to the third order in the velocity expression, and including the observed variable magnetic field (Romanov, Udovichenko, \& Frolov 1987, 1994), is required, if the detected modes are of degree $\ell=2$. On the other hand, the resonance model needs to be further explored. It should be investigated whether nonlinear resonance can also produce a frequency spectrum like the one we observed. At the same time, we will explore the characteristics of the observed frequency spectra, like the phase and the amplitudes of the frequencies, in more detail, to put constraints upon the different rival hypotheses.

New spectroscopic high-resolution observations with a time base of at least 6 weeks are necessary to increase our understanding of the frequency spectrum, especially to confirm the presence or absence of the quintuplet, to obtain a good value of the intensity of the frequency peaks, and to detect the maximum of harmonics of the main frequency. Also, a dedicated observational effort must be done to re-measure the magnetic field of RR Lyrae.

This paper summarizes the results worked out in our article with the same title (Chadid et al. 2000)

Acknowledgments. We wish to congratulate the LOC for the smooth organization of this excellent meeting, and would like to thank the IAU and the SOC for their kind financial support. We also acknowledge for the constructive remarks and useful hints given after our talk.

\section{References}

Aerts, C., De Pauw, M., \& Waelkens, C. 1992, A\&A, 266, 294

Chadid, M. \& Gillet, D. 1997, A\&A, 319, 154

Chadid, M., Kolenberg, K., Aerts, C., \& Gillet, D. 2000, A\&A, in press Cousens, A. 1983, MNRAS, 203, 1171

Cox A. N. 1993, in New Perspectives on Stellar Pulsation and Pulsating Stars, ed. J. M. Nemec \& J. M. Matthews (Cambridge: Cambridge Univ. Press), 409

Kovács, G. 1994, Ann. New York Acad. Sci., 706, 70

Kovács, G. 1995, A\&A, 295, 693

Kurtz, D. W. 1982, MNRAS, 200, 807

Roberts, D. H., Lehár, J., \& Dreher, J. W. 1987, AJ, 93, 968

Romanov, Yu. S., Udovichenko, S. N., \& Frolov M. S. 1987, Sov. Astr. Lett., 13,29

Romanov, Yu. S., Udovichenko, S. N., \& Frolov M. S. 1994, Bul. Spec. Astrophys. Obs., 38, 169

Shibahashi, H. \& Takata, M. 1995, in ASP Conf. Ser. Vol. 83, Astrophysical Applications of Stellar Pulsation, ed. R. S. Stobie \& P. A. Whitelock (San Francisco: ASP), 42 
Shibahashi, H. 2000, in these proceedings, p. 299

Stellingwerf, R. F. 1978, ApJ, 224, 953

Szeidl, B. \& Kolláth, Z. 2000, in these proceedings, p. 281

Van Hoolst, T., Dziembowski, W. A., \& Kawaler, S. D. 1998, MNRAS, 297, 536

Van Hoolst, T. 2000, in these proceedings, p. 307

\section{Discussion}

Pawel Moskalik: I like your work very much. However, I do not think you have discovered any nonradial modes. The side-peaks you find in the power spectrum are a simple mathematical consequence of the amplitude being periodically modulated. Also the asymmetry (different amplitudes of side-peaks) can be easily understood as resulting from the phase being modulated as well.

Katrien Kolenberg: You are correct: we have provided evidence for the presence of amplitude modulation in the spectra. On the other hand, all currently acceptable models to explain this modulation are based on nonradial pulsation components.

Marten van Kerkwijk: Have you looked at the higher moments too? What are the results?

Katrien Kolenberg: As mentioned in my talk, period analyses on the second and third order moment also reveal the nonlinearity of the pulsation, and the multiplet structure, as observed in the first moment. I also refer to our article in press to $\mathrm{A} \& \mathrm{~A}$.

Wojtek Dziembowski: The most urgent issue now is to perform a mode identification from high-resolution line profile variations.

Katrien Kolenberg: This will be the next step in our research plan.

Douglas Gough: Where do we find direct evidence for the nonradial modes?

Katrien Kolenberg: The direct evidence will follow from our application of the nonlinear generalization of the moment method.

Alex Schwarzenberg-Czerny: I warn against using CLEAN for assessing amplitude ratios of pulsations at different frequencies. The only way to obtain undistorted amplitudes in power spectra is with DFT (with modifications of window function trimming allowed). All other methods (so-called nonquadratic methods) of power spectrum estimation must distort it, leading to errors of factors of 2 in the case of CLEAN. 